\title{
Climate normalized spatial patterns of evapotranspiration en- hance the evaluation of hydrological models
}

\author{
Julian Koch ${ }^{1, *}$ Mehmet Cüneyd Demirel ${ }^{2}$ and Simon Stisen ${ }^{1}$
}

1 Geological Survey of Denmark and Greenland (GEUS), Department of Hydrology, Copenhagen, Denmark

2 Istanbul Technical University, Department of Civil Engineering, Istanbul, Turkey

* Correspondence: juko@geus.dk

\begin{abstract}
Spatial pattern-oriented evaluations of distributed hydrological models have contributed towards an improved realism of hydrological simulations. This advancement was supported by the broad range of readily available satellite-based datasets of key hydrological variables, such as evapotranspiration (ET). At larger scale, spatial patterns of ET are often characterized by an underlying climate gradient, and with this study, we argue that gradient dominated patterns may hamper the potential of spatial pattern-oriented evaluation frameworks. We hypothesize that the climate control of spatial patterns of ET overshadows the effect model parameters have on the simulated variability. To solve this limitation, we propose a climate normalization strategy. This is demonstrated for the Senegal River basin as modeling case study, where the dominant north-south precipitation gradient is the main driver of the observed hydrological variability. Two multi-objective calibration experiments investigate the effect of climate normalization. Both calibrations utilize observed discharge $(\mathrm{Q})$ in combination with remote sensing ET data, where one is based on the original ET pattern and the other utilizes the normalized ET pattern. We identify parameter sets that balance the tradeoffs between the two independent observations and find that the calibration using the normalized ET pattern does not compromise the spatial patern performance of the original pattern. However, vice versa, this is not necessarily the case, since the calibration using the original ET pattern showed a poorer performance for the normalized pattern. Both calibrations reached comparable performance of Q. With this study, we identified a general shortcoming of spatial pattern-oriented model evaluations using ET in basins dominated by a climate gradient, but we argue that this also applies to other variables such as, soil moisture or land surface temperature.
\end{abstract}

Keywords: evapotranspiration, spatial patterns, model evaluation, remote sensing, hydrological modeling, climate normalization

\section{Introduction}

The distinct spatial variability of hydrological processes leads to complexity inherent to a range of environmental and societal concerns such as floods, droughts or climate change impacts. To tackle such concerns, spatially distributed modelling is required to support targeted decision making. The integration of satellite remote sensing data marked an important step in the last decade of spatially distributed hydrological model development. Satellite remote sensing provides spatially explicit data that have the potential to effectively improve spatial realism of hydrological simulations through improved parametrization schemes in conjunction with spatial pattern-oriented model evaluation frameworks. With respect to the enhanced parametrization, spatio-temporal vegetation data derived from satellite systems were for example successfully applied to spatially distributed vegetation related parameters, such as root depth or crop coefficient [1-4]. In broader terms, seamless parameter distributions that comprise consistent spatial patterns have proven to overcome pitfalls of spatially distinct, i.e. unit-based, model parametrizations $[5,6]$. The second aspect, namely spatial pattern-oriented model evaluation, has proven to be extremely insightful for diagnosing structural model deficiencies relating to the spatial 
pattern performance $[7,8]$ or as objective functions in multi-variable calibration frameworks [9] which typically combine discharge observations with a remotely sensed variable such as evapotranspiration (ET) [10], land surface temperature (LST) [11] or soil moisture (SM) [12]. It is often found that the temporal model evaluation using discharge is complementary to the spatial pattern evaluation using satellite derived data and that tradeoffs are often very limited[1]. Furthermore, the potential of satellite remote sensing data is also to constrain model calibrations for data scarce regions.

In the recent literature on spatial pattern-oriented model evaluation, we found that the investigated patterns were often spatially linked to distinct climate or landscape characteristics of the study sites. This often resulted in a very satisfying pattern performance, because the dominating processes causing the spatial pattern, i.e. a distinct precipitation gradient, overshadowed the imprint of model parameters on the simulated spatial pattern. For example, SM and ET patterns of the Volta Basin that exhibit a strong north-south gradient caused by a corresponding precipitation gradient led to very satisfying and comparable results for a variety of tested calibration experiments [12]. A similar impression could be obtained from a model evaluation of the U.S using remotely sensed LST data. The LST patterns at this scale are dominated by air temperature, which resulted in correlation coefficients above 0.95 for most of the evaluated LST patterns [13]. LST was also utilized to evaluate the performance of a hydrological model of a Mexican basin with a $2400 \mathrm{~m}$ elevation range [14]. LST obviously followed the topography gradient and a correlation coefficient greater than 0.8 could be attested to most of the evaluated patterns. Moreover, several ET datasets were compared at pan-European scale and at basin scale and it was found that the continental ET patterns were in better agreement with each other than the patterns at basin scale [15]. This is a consequence of the fact that at European scale, the patterns are driven by climate variability, whereas at catchment scale, land cover and soil characteristics emerge as dominant drivers of spatial variability.

With this study we intend to investigate if the effectiveness of a spatial pattern-oriented model evaluation is limited by dominating gradients that are beyond the control of model parameters. We expect the sensitivity of model parameters to be hampered for such patterns and we suggest normalizing the spatial patterns to enhance the sensitivity of the model parameters. The idea of normalization, with the aim to decipher overshadowed hydrological processes, is not novel as such. The evaporative fraction (EF), i.e. actual ET normalized by potential ET, has been linked to SM to diagnose model functioning at larger scale across the U.S. [16,17], which has been utilized to evaluate models but also to gain process understanding. The EF has also been applied to evaluate a catchment model in Denmark [18]. Here, instantaneous ET was normalized by the available energy to remove the effect of diverging energy inputs between the evaluated models. The notion of precipitation normalization is the principal element of the Budyko framework [19], where ET and potential ET are normalized by precipitation, resulting in the evaporation index and the aridity index, respectively. This allows to study the dominance of water or energy limitation of a basin. The Budyko hypothesis has successfully been applied to evaluate hydrological models [20]. The evaporative index has been utilized to discriminate performance of various hydrological models for the Indian sub-continent [21]. The evaluation has been conducted grid-based as well as basin-based and allowed insights into diverging precipitation partitioning across models. In more general terms, the concept of process constraints, defined as expert knowledge based ratios of simulated fluxes and model forcings, has been introduced to enable model evaluation also for ungauged basins [22].

We hypothesize that normalizing spatial patterns of satellite based hydrological observations that exhibit a clear gradient, which is caused by processes not represented in hydrological models, conveys alternative information relevant for calibration. To investigate this research topic, we set up a hydrological model of the Senegal River basin, which is characterized by a distinct north-south gradient in precipitation. This gradient is the dominant 
driver of the spatial variability of hydrological fluxes of the basin. We focus on discharge observations in combination with spatial ET patterns as target variables for the model evaluation. In this context, we suggest a novel normalization method of the ET pattern that utilizes a fitted polynomial trend relating the observed ET to precipitation. Through two calibration experiments we intend to explore the implications of calibrating a hydrological model against a normalized pattern of ET.

\section{Materials and Methods}

\subsection{Study Site}

The study was carried out in the $350,000 \mathrm{~km}^{2}$ large Senegal River, which is the second largest basin in West Africa (Figure 1). The Senegal River spans four riparian states, namely Guinea, Mali, Senegal and Mauritania, and extends over diverse hydroclimatic conditions from arid climate in the northern Sahel zone to tropical climate in the southern Guinean zone. The distinct North-South precipitation gradient ranges between $200 \mathrm{~mm}$ $\mathrm{yr}^{-1}$ to over $1500 \mathrm{~mm} \mathrm{yr}^{-1}$ with clear implications for the hydrology of the basin. Almost $50 \%$ of the generated discharge observed at the Bakel station originates from the southern Dakka sub-catchment which covers only approximately $5 \%$ of the Bakel drainage area [23]. Besides the distinct spatial variability, the temporal flow regime follows a clear seasonality that is characterized by a 6-month rainy season that has its onset in June. Approximately $95 \%$ of the annual precipitation of the Senegal River $\left(600 \mathrm{~mm} \mathrm{yr}^{-1}\right)$ occurs in the months June till October. Peak flows at the Bakel station range between $2000 \mathrm{~m}^{3} \mathrm{~s}^{-1}$ and $4000 \mathrm{~m}^{3} \mathrm{~s}^{-1}$ and typically occur in the months August and September. The Manantali dam, just downstream of the Makana station is the largest reservoir of the Senegal River basin and artificializes the downstream flow regime [24]. The four stations that are located upstream of major hydraulic infrastructures, where close-to natural flow conditions can be assumed, are Qualia, Gourbassa, Dakka and Makana.

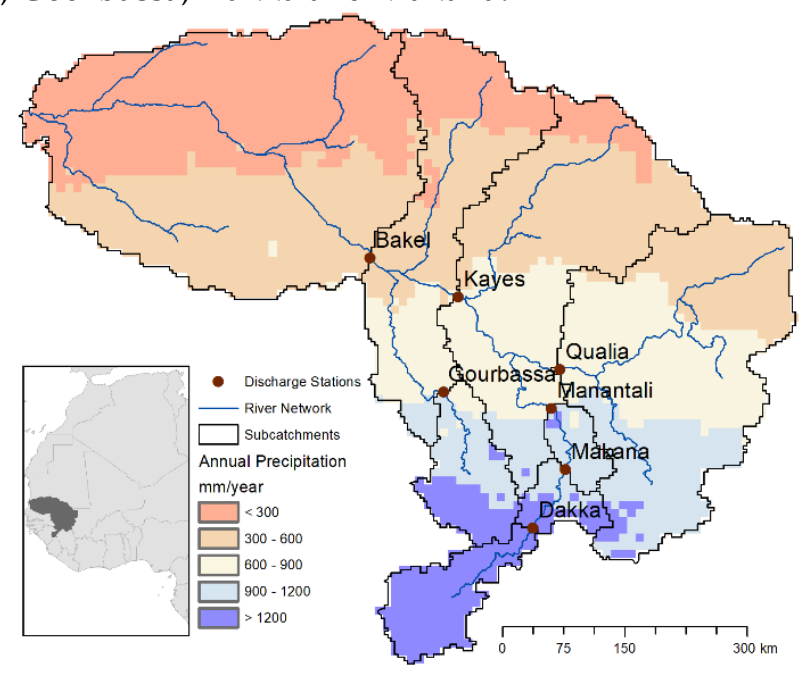

Figure 1. The Senegal River basin delineated as eight sub-catchments. The seven sub-catchments with available discharge records are stated by station names. The long-term average annual precipitation is based on CHIPRS 2.0 for the period of 1990-2019. The overview figure depicts the location of the Senegal River in Western Africa.

\subsection{Hydrological Model}

We applied the mesoscale Hydrological Model (mHM) v.11.1 [25] to model the hydrological cycle of the Senegal River basin. $\mathrm{mHM}$ is a spatially distributed, grid-based, conceptual hydrological model that was specifically developed for providing physically meaningful spatially explicit predictions of hydrological variables through a multiscale parameter regionalization framework (MPR) [26-28]. Canopy interception, soil moisture dynamics, infiltration, surface runoff, discharge generation, evapotranspiration, subsurface storage, deep percolation, baseflow, and river routing are among the processes that 
are accounted for in mHM. The MPR framework consolidates model input data across spatial scales, i.e. high resolution geophysical basin characteristics (e.g. elevation, soil texture or vegetation) and coarse resolution meteorological forcing (e.g. precipitation, temperature and potential evapotranspiration). The hydrological model routines that generate output are executed at an intermediate scale that lies in between the high-resolution basin characteristics and the coarse meteorological forcing. Model parameters are regionalized through simple and well-established transfer functions linking the spatially distributed basin characteristics to seamless parameter fields. The transfer functions are controlled via global parameters which allows for efficient model calibration with a limited number of calibration parameters. It has also been documented that $\mathrm{mHM}$ parameters are scale independent, which allows the model to be calibrated computationally efficient at coarse resolution and subsequently to be applied at high spatial resolution with the same parameters [29]. To further improve realism of spatially distributed model output, two transfer functions have recently been implemented in mHM [1]. The first links fully distributed vegetation characteristics, i.e. remotely sensed Leaf Area Index (LAI), to a spatially distributed crop coefficient that is used for scaling the potential evapotranspiration (PET) to account for heterogeneous landcover. The second utilizes spatially distributed soil texture information to derive a field capacity dependent spatially varying root depth parameter. Both transfer functions are essential for the effective integration of satellite based observations, of e.g. evapotranspiration, through a spatial pattern oriented calibration framework aiming at increasing realism of the spatially distributed model simulations $[1,10]$.

mHM has successfully been applied for several West African basins using a variety of forcing data and observational data as calibration targets [12,30-32]. In our model setup for the Senegal River basin, elevation was retrieved from the Shuttle Radar Topographic Mission. While available in multiple resolutions, the $500 \mathrm{~m}$ resolution was used for the purpose of this study. The required slope, aspect, flow direction and flow accumulation data were derived from this elevation dataset. Soil texture variables, clay content, sand content and bulk density, were derived from the AfSoilGrids250m dataset [33]. The variables are available at $250 \mathrm{~m}$ resolution for 6 layers with varying depths covering the top $200 \mathrm{~cm}$ and were resampled to $500 \mathrm{~m}$. Annual land use maps for the Senegal River were generated based on the MODIS' MCD12Q1.006 product, which has a native resolution of $500 \mathrm{~m}$ [34]. Land use was reclassified into three classes, namely permeable, impermeable and forest. Long-term monthly LAI climatologies used to calculate a spatio-temporally varying crop coefficient were based on MODIS' MCD15A2H.006 product [35]. The original 8-day composite dataset was aggregated to long-term monthly means at a spatial resolution of $500 \mathrm{~m}$. Daily averaged air temperature data were processed based on the ERA5 reanalysis dataset at $0.1^{\circ}$ resolution [36]. PET was estimated based on the HargreavesSamani [37] model using ERA-5 air temperature data (daily min, max and mean) as input. For this purpose, the PyETo Python library (https://pyeto.readthedocs.io) was applied. Daily gridded precipitation data was obtained from the Climate Hazards Group InfraRed Precipitation with Stations (CHIRPS) 2.0 dataset, which has a native resolution of $0.05^{\circ}$ $[38,39]$. For the Senegal River basin mHM model the following spatial resolutions have been defined: $500 \mathrm{~m}$ for the basin characteristics, $5 \mathrm{~km}$ for the intermediate modeling resolution and $10 \mathrm{~km}$ for the meteorological forcing. A 16-year period (1996-2011) with a 2year warming period was simulated.

\subsection{Observational Data}

The model performance was evaluated against two independent hydrological fluxes, namely discharge $(\mathrm{Q})$ and evapotranspiration (ET). Continuous daily $\mathrm{Q}$ observations were available from 1996 to 2005 at seven stations in the Senegal River basin (Figure 1), three of which are located downstream of major dams (Bakel, Kayes and Manantali). Hence, the flow regimes are heavily altered and since mHM does not represent the effect of dams and only simulates natural flow conditions, the three stations were excluded from the model evaluation. 
Spatial patterns of ET were derived from MODIS' MOD16A2.006 product [40], referred to as MOD16 hereafter. The ET data were processed for the period 2002 to 2011 for Terra and Aqua satellite systems. First, average monthly maps were calculated taking only high-quality data into account and subsequently, monthly climatologies were calculated by averaging each month over the 10 year period. Since the mHM model was executed at spatial resolution of $5 \mathrm{~km}$, the MOD16's native resolution of $500 \mathrm{~m}$ was resampled to $5 \mathrm{~km}$ using the mean function. Due to expected large uncertainties of ET over lakes and urban areas, the two land cover classes were removed from the ET dataset. Finally, the monthly climatologies were aggregated to rainy- and dry-season, where the rainy season was defined as the 6-month period of June till November. Based on MOD16, the monthly basin average ET of the 6 rainy season months is above $10 \mathrm{~mm} \mathrm{mth}^{-1}$ with a peak in August and September of approximately $65 \mathrm{~mm} \mathrm{mth}^{-1}$. The purpose of aggregating the ET data in time to a rainy season climatology was to obtain a robust pattern that is informative for model calibration. It is expected that the climatology is more sensitive to calibration parameters of the hydrological model, whereas ET snapshots at daily or weekly scale are more linked to climate variability. Similar processing has been reported for related spatial pattern-oriented calibration studies [3].

\subsection{Model Evaluation}

The model performance was evaluated using two multi-component objective functions. For the temporal evaluation of simulated Q, the Kling-Gupta-Efficiency (KGE) was applied [41]:

$K G E=1-\sqrt{(r-1)^{2}+(\alpha-1)^{2}+(\beta-1)^{2}}$,

where $r$ is the Pearson correlation coefficient between observed and simulated $\mathrm{Q}, \alpha$ is the standard deviation fraction of observed and simulated $Q$ and $\beta$ is the bias fraction relating the observed and simulated average $\mathrm{Q}$.

For the spatial pattern evaluation of simulated ET, the Spatial Efficiency metric (SPAEF) was utilized $[1,10]$ :

$S P A E F=1-\sqrt{(r-1)^{2}+(\alpha-1)^{2}+(\gamma-1)^{2}}$,

where $r$ is the Pearson correlation coefficient between observed and simulated spatial patterns of ET, $\alpha$ is the coefficient of determination fraction of observed and simulated ET and $\gamma$ quantifies the fraction of the histogram intersection based on the z-scores of observed and simulated ET. The three SPAEF components are bias-insensitive, which is an important consideration for a spatial pattern-oriented model evaluation. The magnitude of the observed spatial patterns may be uncertain, but the data contain valuable spatial pattern information. Both, KGE and SPAEF have a range from $-\infty$ to the optimum of 1.

\subsection{Climate Normalization}

We introduce a novel climate normalization framework that aims at removing the precipitation induced trend that is evident in the spatial patterns of ET in the Senegal River. In principle, the framework is generic and applicable for alternative variables as well, for example trends in any spatially distributed hydrological variable driven by precipitation, temperature, etc. can be analyzed. We formulate a simple function to calculate the normalized spatial pattern of ET (ET norm $)$ by subtracting the precipitation induced trend $(f$ (Precip) $)$ from the ET observation:

$E T_{\text {norm }}=E T-f($ Precip $)$,

For the trend function we fitted a $4^{\text {th }}$ degree polynomial function with truncated tails to model ET based on precipitation as the only input. The method is illustrated in Figure 2, where the North-South precipitation gradient that causes the ET trend is apparent. The 
upper tail of the ET and precipitation relationship is flat, suggesting energy limited conditions. The high ET values found for low precipitation values are located in the western coastal region of the Senegal River basin and are likely caused by intensive irrigation activities in the delta. The effect of climate normalization becomes apparent in Figure 2, the original ET pattern is a direct imprint of the precipitation pattern, whereas the normalized pattern conveys more complexity of the hydrological processes taking place in the basin. The normalized pattern is normally distributed with a mean of zero and captures the deviation of the original ET pattern to the fitted trend model. Positive values are partly correlated with a higher clay content, due to the better water-holding capacity of clayey soils and LAI is found to be higher for positive values of normalized ET (not shown).

For the spatial pattern evaluation of the simulated normalized ET we employed the same trend function as applied to normalize observed ET. To accommodate any potential bias between the observed ET and the hydrological model, the ET bias of the model is added to the trend function before the normalization is applied.
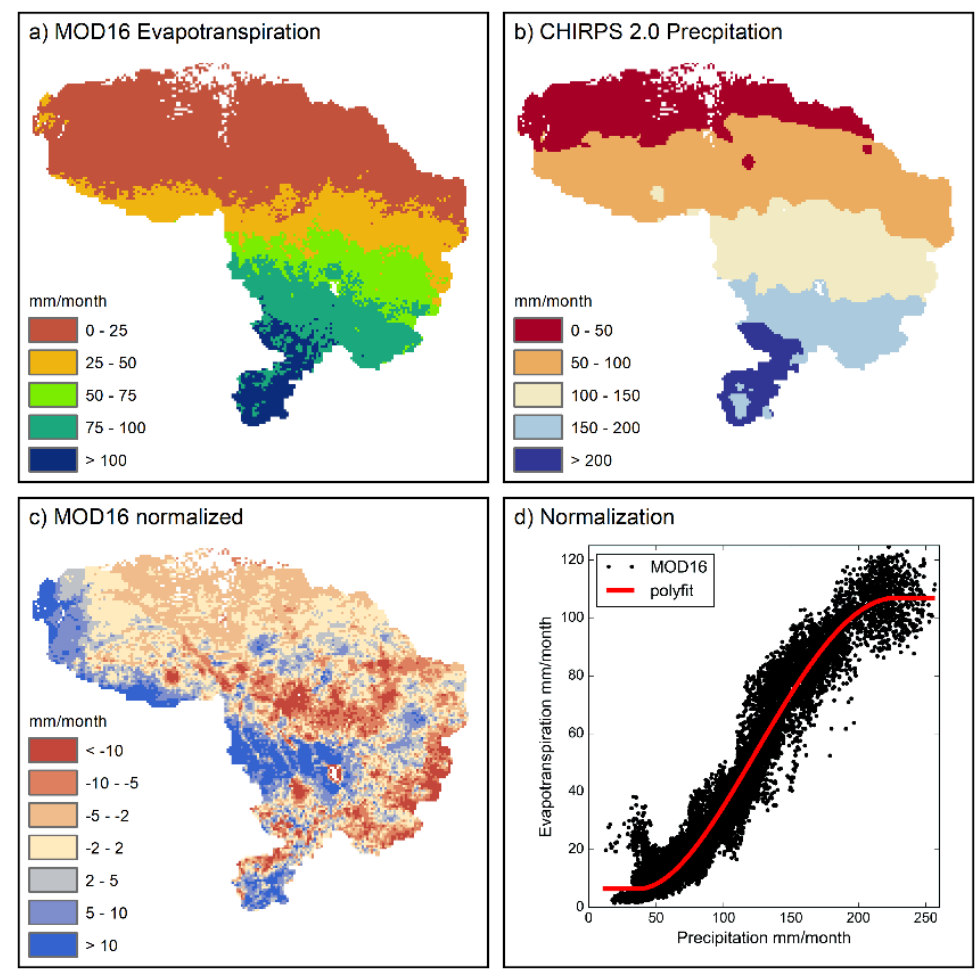

Figure 2. Panel a) depicts the long-term (2002-2011) average monthly rainy-season (June-November) evapotranspiration based on MOD16A2.006. Panel b) shows the long-term average monthly rainy-season (June-November) precipitation based on CHIPRS 2.0. Panel c) presents the precipitation normalized rainy-season evapotranspiration. The fitted polynomial model applied for the normalization is shown in panel $\mathrm{d}$ ).

\subsection{Calibration Experiments}

Two calibration experiments were performed with the model-independent Ostrich optimization software [42] using the ParaPADDS search algorithm. ParaPADDS is a parallelized multi-objective Dynamically Dimension Search (DDS) [43] algorithm that identifies a Pareto front of non-dominated optimal solutions, which is particularly useful for multi-objective calibrations. The ParaPADDS algorithm was run with the user settings of 2000 iterations, with 3 parallel runs, a 0.2 perturbation value and the ExactHyperVolumeContribution as the selection metric. In this study, two objective functions (OFs) were used for the model calibration. Ostrich minimizes any given OF and since KGE and SPAEF have an optimal value of 1 we calculated the squared residuals for each of the two OFs.

$S R_{E T}=(1-S P A E F)^{2}$, 
where $S R_{E T}$ represents the squared residuals for the long-term average rainy season ET model performance applying SPAEF as OF. For Q, KGE was calculated at four stations and here we used the sum of squared residuals.

$S S R_{Q}=\sum_{i}^{4}\left(1-K G E_{i}\right)^{2}$,

where $S S R_{Q}$ represents the sum of squared residuals for the $Q$ performance at four discharge stations using KGE as OF.

We have designed two calibration experiments to investigate the potential benefits of climate normalization. Both calibrations used $S S R_{Q}$ as objective function. As additional objective function Cal1 used $S R_{E T}$ based on the original rainy season ET climatology, whereas Cal2 used $S R_{E T}$ based on the normalized rainy season ET climatology. In the SPAEF formulation, $\alpha$ is the coefficient of determination fraction. To avoid division by zero for the coefficient of determination, SPAEF for the normalized patterns was based on the fraction of standard deviations for the $\alpha$ component, as opposed to the fraction of coefficients of determination (Eq 2). This is justifiable, because normalization will result in spatial patterns with a mean of zero.

Prior to calibration, we conducted a comprehensive sensitivity analysis to select the calibration parameters. The sensitivity analysis was based on a one-at-a-time (OAT) perturbation of model parameters and the change in OF was recorded to assess sensitivity. In order to overcome the limitation of the OAT perturbation, which only captures local sensitivity, we designed an ensemble approach taking multiple initial parameter sets into consideration [44]. We selected 8 behavioral initial parameter sets for the OAT sensitivity analysis, i.e. KGE for all $4 \mathrm{Q}$ stations and SPAEF for the original ET pattern greater than 0.33 as well as a positive SPAEF for the normalized ET pattern. The behavioral parameter sets were obtained from a Latin Hypercube Sampling (LHS) of 300 initial parameter sets.

\section{Results}

\subsection{Sensitivity Analysis}

The results of the sensitivity analysis are based on the 8 behavioral parameter sets that were employed in a local OAT sensitivity analysis. The sensitivity for a given parameter perturbation was recorded for the three defined OFs, namely KGE at four discharge stations, and SPAEF for the original and the normalized rainy season ET pattern. Each set of sensitivities was normalized per OF by the maximum to range between 0 and 1 . In total, 21 out of the 45 parameters were selected for subsequent model calibration and the sensitivities for those parameters are shown in Figure 3.

With respect to the different OFs, different parameters were identified as sensitive. Discharge is controlled by the root fraction parameter for forest (rotfrcoffore) and the soil type dependent root fraction parameter (rotfrcofcl). These parameters control the vertical distribution of roots and thereby they affect the water balance, i.e. the partitioning into soil water available for transpiration and runoff generation. Furthermore, two pedo transfer function parameters that control the saturated water content (ptflowconst and ptflow db) are identified as being sensitive for the simulation of discharge. The spatial pattern of ET is sensitive to the root fraction parameters as well as for the PET scaling function that estimates a spatially distributed crop coefficient. Most sensitive parameters are the maximum and minimum of the crop coefficient (pet_b and pet_a, respectively) as well as the shape of the exponential function (pet_c). The sensitivities for the normalized ET pattern diverge with respect to the original ET pattern. Here, the pedo transfer parameters as well as the PET scaling function are more sensitive and the forest root fraction (rotfrcoffore) is less sensitive. The variability of the ensemble is apparent for some, but not for all parameters. For example, the soil type dependent root fraction parameter (rotfrcofcl) is the most sensitive parameter for all 8 initial parameter sets for the normalized ET patterns, whereas the sensitive for forest root fraction (rotfrcoffore) parameter varies between the 8 initial parameter sets for SPAEF calculated for the original ET patterns. 


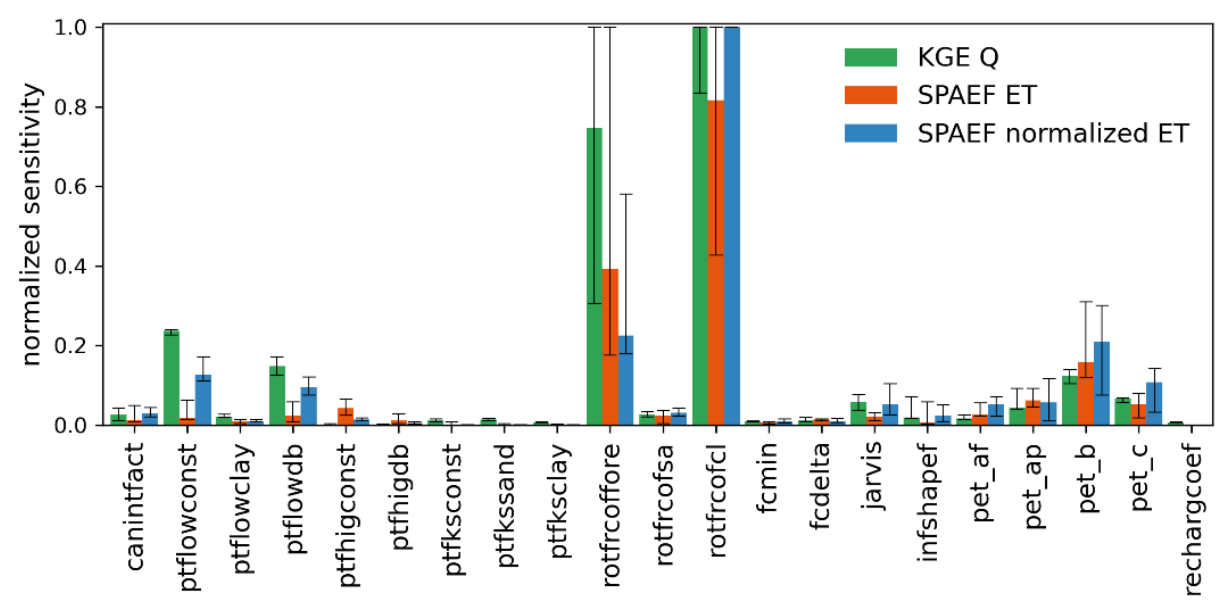

Figure 3. Results of the sensitivity analysis. The normalized sensitivity is given for the 21 parameters that were selected for subsequent model calibration. Sensitivity with respect to three objective functions is shown for the median of an ensemble of 8 different behavioral parameter sets. The whiskers represent the upper and lower limits of the inter-quantile range.

\subsection{Pareto Front}

The results of the two calibration experiments are presented in Figure 4. Each calibration comprised 2000 model runs and the non-dominated runs, which mark the pareto front, were identified by Ostrich. The characteristic of a non-dominated run is that one OF cannot be improved without compromising the other. Ostrich does not provide the user with an optimal run, instead it is up to the user to study the tradeoff between the applied OFs to select a parameter set or an ensemble for subsequent model application. The distribution of runs along the pareto front contains an element of randomness as it is a result of the ParaPADDS search algorithm. Both calibrations depict a clear pareto front describing the tradeoff between the temporal discharge performance and the spatial ET performance. The SPAEF residuals for the normalized ET patterns are larger than for the original ET patterns which indicates that the simulated patterns after normalization are more dissimilar to the normalized target pattern than the original ET patterns. Opposed to this, the discharge performance is comparable between the two calibrations.

In order to investigate the two calibration experiments in more detail, we have selected a subset of the non-dominated runs. This subset contained balanced runs which are located around the optimum disregarding the tails of the pareto front which focus solely on a single OF. This selection results in what is referred to as the balanced sets in Figure 4 $a$ and $b$. For the selection, the tail of high KGE residuals was truncated by the median KGE plus one standard deviation and the for the SPAEF residuals, values that exceed median SPAEF plus three standard deviations were excluded. For each calibration, a single run was selected as featured run in order to visualize simulation results. 



Figure 4. Calibration results for Cal1 (top panel) and Cal2 (bottom panel). Each multi-objective calibration features 2000 runs (gray) of which the non-dominaed runs (orange) were identified. The balanced runs (blue) represent an ensemble that is located around the pareto optimum. A single featured run (green) for both calibrations was selected to present simulation results.

For cal1 the amount of selected balanced runs was 28 and for cal2 42 runs were selected as balanced runs. The deviation is simply a result of how ParaPADDS samples the parameter space and should not be understood as a degree of robustness of the two calibrations. Table 1 provides an overview of the median and standard deviation of the applied OFs computed for the balanced runs of cal1 and cal2. Discharge performance is comparable with a slight advantage of cal1. Spatial pattern performance of ET, expressed as SPAEF, is also comparable between the calibrations even though cal2 is not explicitly calibrated against the original ET patterns. Differences between the calibrations become apparent for the normalized ET performance. Here cal2, which is calibrated against the normalized patterns stands out with an improvement in SPAEF by 0.1 with respect to cal1. The three SPAEF components are also given in table 1. For the original patterns, correlation is the dominating component which is close to optimal in both calibrations. Here, the variability component was simulated poorest by both calibrations. For the normalized ET evaluation, the scores of the individual SPAEF components varies more distinctly. In this case the variability component was simulated best by both calibrations, where as the correlation has the lowest scores and also the most apparent difference between cal1 and cal2.

Table 1. Summary of the calibration results taking only the balanced runs (Figure 4) into consideration. The number of runs that are contained in the ensemble varies between the two calibrations and median and standard deviation (std) of the ensembles are presented. The three components of the SPAEF are stated: Correlation $(r)$, variability $(\alpha)$ and distribution $(\beta)$.

\begin{tabular}{c|c|c|cccc|rrrr|}
\multicolumn{2}{c}{} & Q & \multicolumn{4}{c|}{ ET } & \multicolumn{4}{c|}{ normalized ET } \\
\cline { 3 - 10 } \multicolumn{2}{c|}{} & KGE & SPAEF & r & $\alpha$ & $\beta$ & SPAEF & r & $\alpha$ \\
\hline Cal1 & median & 0.73 & 0.49 & 0.97 & 0.56 & 0.74 & 0.23 & 0.28 & 1.07 & 0.75 \\
n=28 & std & 0.05 & 0.02 & 0.00 & 0.01 & 0.01 & 0.08 & 0.05 & 0.12 & 0.04 \\
\hline Cal2 & median & 0.70 & 0.52 & 0.97 & 0.58 & 0.75 & 0.33 & 0.37 & 0.99 & 0.78 \\
n=42 & std & 0.05 & 0.02 & 0.00 & 0.01 & 0.01 & 0.05 & 0.04 & 0.07 & 0.02
\end{tabular}


In order to further evaluate the simulated pattern of ET, Figure 5. The spatial pattern evaluation of the balanced runs of Cal1 and Cal2 against the rainy-season ET climatology (panel a)) and the precipitation detrended climatology (panel b)). Scores for SPAEF and its three components are presented. The boxplots show the median (black line), interquantile range (box) and 1st and 99th percentiles (whiskers). Figure 5 elucidates the statistics provided in Table 1 in more detail. The balanced runs of cal1 and cal 2 are crossevaluated, i.e. the balanced runs obtained from cal2 are also evaluated against the original ET patterns using SPAEF. Both calibrations show similar performance for the original ET pattern performance, in spite of the fact that cal2 is only calibrated against the normalized pattern. The correlation component is close to one with a minimal variability. Call and cal2 show similar performance based on all three SPAEF components for the evaluation of the original ET pattern. For the normalized ET evaluation, cal2 finds more accurate solutions (i.e. closer to 1) than cal1. This results in an overall increase in SPAEF. Opposed to the original ET pattern evaluation, the normalized evaluation is less dominated by the correlation term and the inter-quantile range for each metric of the balanced runs is also larger.
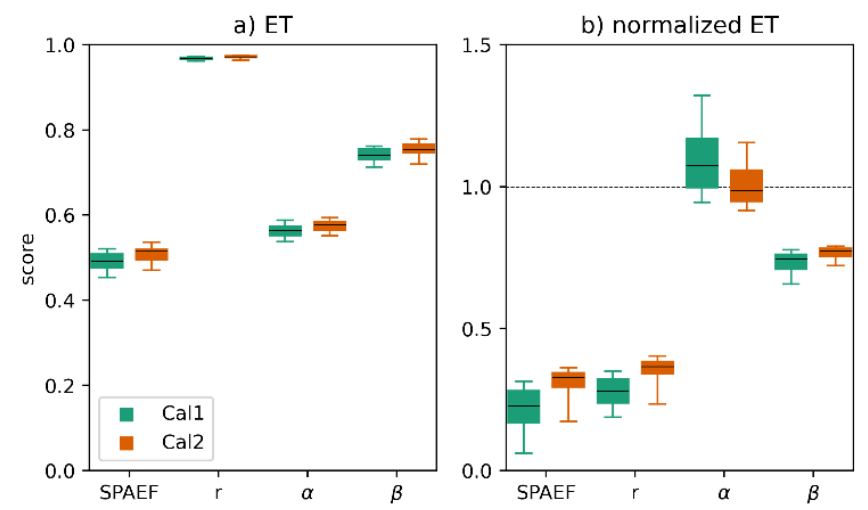

Figure 5. The spatial pattern evaluation of the balanced runs of Cal1 and Cal2 against the rainyseason ET climatology (panel a)) and the precipitation detrended climatology (panel b)). Scores for SPAEF and its three components are presented. The boxplots show the median (black line), interquantile range (box) and $1^{\text {st }}$ and $99^{\text {th }}$ percentiles (whiskers).

The climate normalization of the simulated ET data is visualized in Figure 6. The proposed normalization framework accepts any potential bias inherent to the simulation. Thus, the polynomial model fitted to the observed ET data is shifted, taking into account the simulation bias. In our case, the model has a positive bias of around $30 \mathrm{~mm} \mathrm{mth} \mathrm{m}^{-1}$. The simulation captures the flattening of the relationship between precipitation and ET once energy-limited conditions are present. The observed flattening at the dry end, which is evident in the MOD16 data, is not captured accordingly by the model and instead simulated ET continuous to decrease with decreasing precipitation. Also, the high ET rates found at the dry end of the plot in Figure 2, which can be attributed to irrigation, are not captured since irrigation is not among the described processes in mHM. Both, Table 1 and Figure 5 underline that the variability component is captured quite accurately by cal1 and cal2 for the normalized pattern. Basically, this component describes the deviation from the trend line and by taking the model bias into account, the variability can be adequately matched by the model despite any potential bias. 


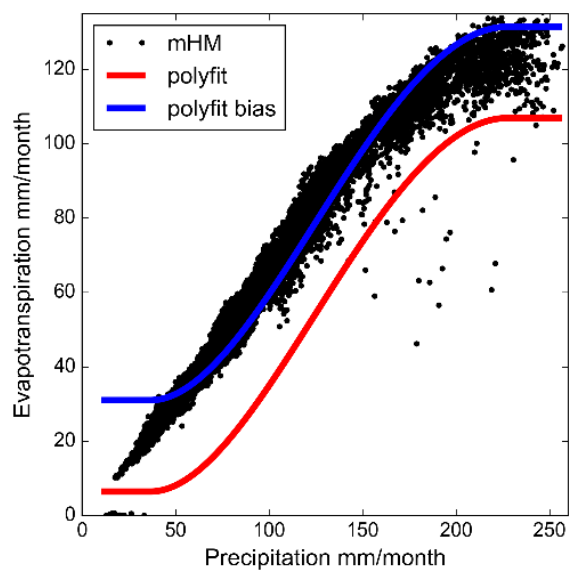

Figure 6. Example of simulated ET (mHM) and the applied precipitation based detrending method. The fitted polynomial using observed ET (red) is shifted to account for the model bias (blue).

The simulated spatial patterns of ET obtained from the balanced runs from call and cal2 with respect to the observed rainy season pattern (MOD16) are shown in Figure 67. Despite of the notable bias, the original pattern of cal1 and cal2 both reflect the northsouth trend accordingly. The SPAEF-based evaluation attests a slightly better pattern performance to cal2 than cal1. The normalized rainy season ET pattern contains more complexity, and it becomes obviously more challenging to achieve a satisfying pattern performance. Based on the SPAEF evaluation cal2 is more accurate than cal1 in capturing the normalized ET pattern. Visually, there lacks resemblance between the observed normalized pattern for both calibrations. Some similarity is notable, e.g. negative values in the northern part, central part and eastern part of the Senegal River basin. However, many details of the spatial pattern are not captured by the model.

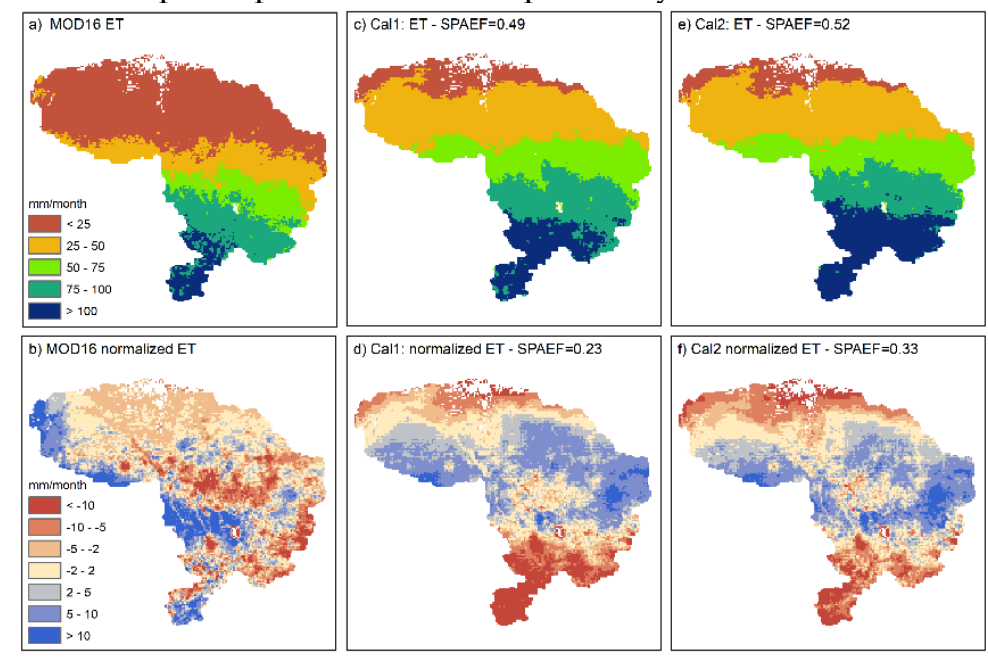

Figure 7. Reference ET (MOD16) (a) and b)) and simulation results (c) -f)) of the featured runs of Cal1 and Cal2. Top row presents the long-term rainy-season ET climatology whereas the bottom row depicts the precipitation detrended climatology. The spatial pattern evaluation (SPAEF) with respect to the reference is given for the panels shown simulation results $(c)-f)$ ).

\subsection{Discharge Evaluation}

Figure 8 presents the discharge evaluation at the four gauging stations using continuous daily observations over a 10-year period. The simulated $\mathrm{Q}$ was obtained from the featured runs (Figure 4) and in terms of KGE, cal1 tends to perform better or at least as good as cal2 at all four stations. The Gourbassa station obtained the highest KGE scores for both calibration experiments, whereas the Dakka stations showed the poorest results. For the latter, the model failed to reproduce the delayed flows towards the end of the 
rainy season. Overall, interannual variability is captured by the model since magnitude of the high flows follows the observations.

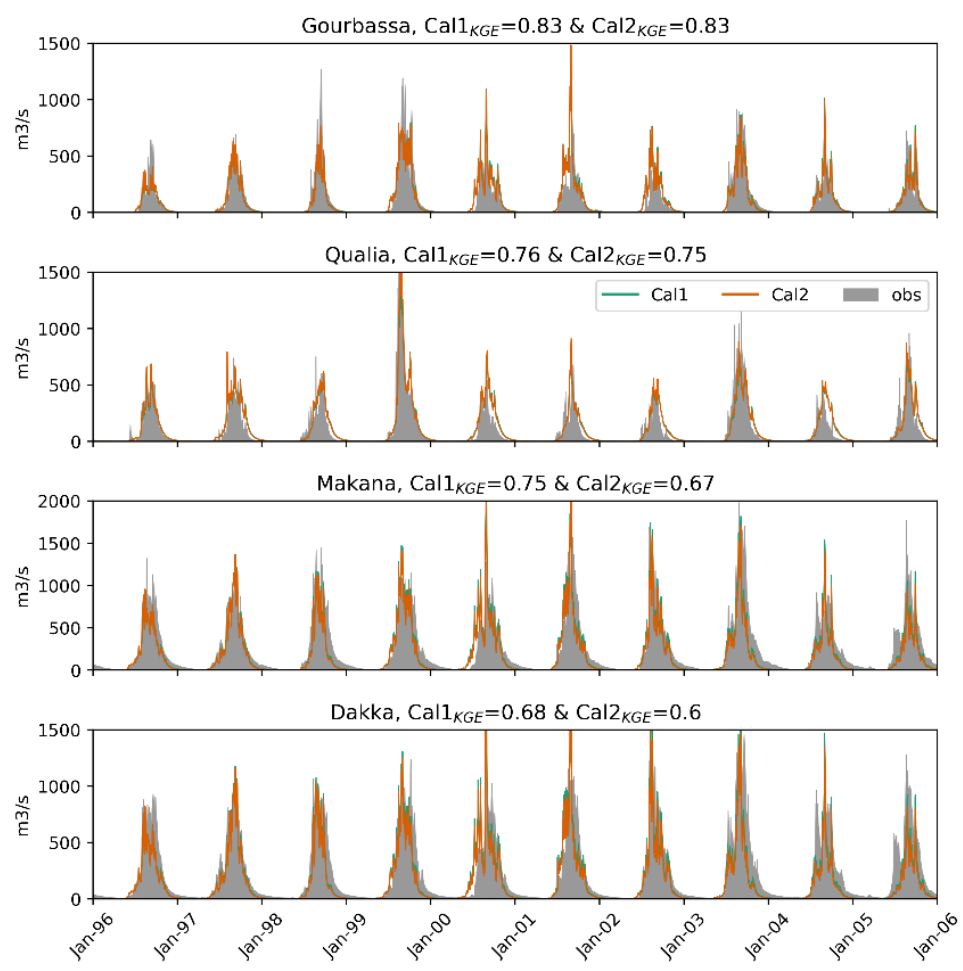

Figure 8. Simulation results of the featured runs of Cal1 and Cal2. Discharge is evaluated for the entire 10-year simulation period at the 4 stations used in calibration. The KGE scores are given in the title of each panel.

\section{Discussion}

\subsection{Normalization Method}

The proposed grid-based normalization method is based on a fitted polynomial trend model. The deviation of ET with respect to the trend model provides information on to what extent ET, at a given location with a certain precipitation input, is higher or lower than an expected ET flux derived from the trend model. In principle, this information is expected to relate to physical basin properties, i.e. better water holding capacity in clayey soils resulting in positive normalized values and sparse vegetation resulting in negative normalized values as a consequence of reduced transpiration and increased surface runoff or recharge. This process heterogeneity can be related to model parameters that are optimizable through calibration. An alternative precipitation normalization strategy is to simply calculate the evaporation index by dividing ET with precipitation [21]. This index provides information on the degree of water limitation and precipitation partitioning of a grid cell, but does not reflect any potential non-linearity contained in the relationship between ET and precipitation. Further, a potential bias in the simulation may pose a challenge in the calculation and comparison between an observed and a simulated evaporation index.

In the proposed normalization, the simulation bias is taken into account by shifting the trend model up or down based on the sign and magnitude of the bias. This works effectively (Figure 6) and falls in line with the general notion of bias-insensitivity for spatial pattern-oriented model evaluations [10,13]. A downside of the normalization strategy is that manual fitting of the polynomial trend model is required. This may pose problems when calibrating multiple basins, utilizing alternative ET sources, or just evaluating a different time period, because an individual trend model has to be fitted for each normalization. 
An advantage of the proposed normalization method is that, in principle, the method is applicable for different variables causing a gradient associated to an observed spatial pattern. This could for example be PET or air temperature when evaluating the spatial pattern performance of a large-scale basin with a distinct climate gradient or a smaller basin with a distinct topography gradient. The topography and air temperature normalization would especially be relevant for LST evaluations whereas PET normalization would be useful for ET evaluations. We consider normalization of remotely sensed SM as an alternative prospect since gradients in SM, just like ET, are often controlled by precipitation or other climate variables[12]. In basins with more complex gradients, multi-variate trend models could also be an option for the normalization.

The usefulness of a normalized spatial pattern of a remotely sensed hydrological variable will also depend on the quality of the data. In this study, the quality of the precipitation data is essential, and precipitation uncertainties may introduce errors in the normalized pattern which hampers the calibration. Nevertheless, the precipitation utilized in the normalization is the same as being used as forcing in the hydrological model. Therefore, artefacts in the normalization caused by erroneous precipitation data may be compensated by the hydrological model which uses the same data as input.

The Senegal River basin, with its distinct north-south climate gradient, was a perfect testbed for developing the proposed normalization technique. Future research should investigate basins with gradients driven by alternative processes.

\subsection{Calibration Strategy}

The Pareto fronts obtained by the ParaPADDS algorithm showed well-defined tradeoffs between $\mathrm{Q}$ and ET performance for both calibration experiments. In theory, all Pareto optimal solutions (non-dominated) can be considered candidates for the "best fit" parameter set. In this study, we tried to avoid identifying a single run as the optimal solution, although "featured runs" were selected for visualization purposes. Instead, we focused on selecting a subset of the full Pareto front containing balanced solutions. This subset was treated as an ensemble and the statistics of the OFs were subsequently analyzed.

The multi-component SPAEF metric allows to interpret the spatial pattern performance of a model in more detail. For example, we found that cal1 was largely dominated by correlation, because the allocation of high and low ET was constrained by the precipitation input and thus, the effect of model parameters was largely overshadowed. The correlation component of SPAEF in cal2 was largely reduced since the normalized pattern was not controlled by the precipitation forcing and instead the effect of model parameters is expected to be more prominent. The $Q$ performance can be regarded as comparable between the two calibration experiments, even though cal1 is slightly in favor with respected to the balanced runs (Table 1). It was not anticipated that the balanced runs of cal2 resulted in a better spatial pattern performance for the original ET pattern than cal1. However, the slightly reduced $Q$ performance of the balanced runs of cal2 may explain this. In general terms, this may also relate to the way the balanced runs were selected and how the calibration evolved.

The robustness of the calibration experiments and the effectiveness of the proposed normalization strategy should be investigated in more detail. Ideally by means of an ensemble approach containing different precipitation forcing data sources as well as an ensemble of ET references [31,32].

Even though the improvement with respect to the normalized pattern performance of cal2 (SPAEF=0.33) in comparison to cal1 (SPAEF=0.23) is clear, the visual assessment of the featured runs in Figure 7 is difficult to interpret. The normalized pattern from cal2 possesses more variability, especially in the southern part of the Senegal River basin, which resulted in an improved SPAEF score with respect to call. The incapacity of the model to further improve the normalized ET pattern performance through calibration may related to the quality of the input data, i.e. spatially distributed soil data or vegetation data, or a limited flexibility of the applied parametrization scheme. Moreover, the 
reference ET dataset, which was derived from MODIS for the purpose of this study, may be inadequate and investigating alternative sources of ET could be an additional way to proceed.

\title{
5. Conclusions
}

Based on recent advances of spatial pattern-oriented model evaluations utilizing satellite remote sensing data, we have identified a shortcoming which holds for basins dominated by a strong climate gradient. We hypothesize that such dominant gradients overshadow the effect of model parameters on the simulated spatial patterns which can hamper the capabilities of model calibration. To investigate this, we conduct two calibration experiments using remotely sensed ET patterns in an original and precipitation normalized form as objective functions and we can draw the following main conclusions from our work:

1) The multi-objective calibration of the Senegal River basin revealed a well-defined Pareto front allowing to investigate tradeoffs between the temporal $Q$ performance at four stations and the spatial pattern performance for long-term average rainy-season ET.

2) Climate normalizing of ET was successfully implemented using a polynomial trend model linking the distinct north-south gradient in precipitation to the spatial pattern of ET.

3) The sensitivity of model parameters varied between the original ET pattern and the normalized ET pattern which supported the conclusion that the normalized pattern contained alternative information.

4) Calibrating against climate normalized patterns did not compromise the performance of the original patterns. Instead, a very comparable performance was obtain between the two calibration experiments.

\begin{abstract}
Author Contributions: Conceptualization, all authors; methodology, J.K. and M.C.D..; software, J.K. and M.C.D.; formal analysis, J.K.; data curation, J.K.; writing-original draft preparation, all authors.; writing - review and editing, all authors.; visualization, J.K.; project administration, J.K.; funding acquisition, J.K.. All authors have read and agreed to the published version of the manuscript.
\end{abstract}

Funding: This research was funded by the Geocenter Denmark under the project "All-WET".

Data Availability Statement: Data, scripts and model setups will be made available and shared upon request to the corresponding author.

Acknowledgments: In this section, you can acknowledge any support given which is not covered by the author contribution or funding sections.

Conflicts of Interest: The authors declare no conflict of interest.

\section{References}

1. Demirel, M.C.; Mai, J.; Mendiguren, G.; Koch, J.; Samaniego, L.; Stisen, S. Combining satellite data and appropriate objective functions for improved spatial pattern performance of a distributed hydrologic model. Hydrol. Earth Syst. Sci. 2018, doi:10.5194/hess-22-1299-2018.

2. Mendiguren, G.; Koch, J.; Stisen, S. Spatial pattern evaluation of a calibrated national hydrological model - a remote-sensingbased diagnostic approach. Hydrol. Earth Syst. Sci. 2017, 21, 5987-6005, doi:10.5194/hess-21-5987-2017.

3. Soltani, M.; Bjerre, E.; Koch, J.; Stisen, S. Integrating remote sensing data in optimization of a national water resources model to improve the spatial pattern performance of evapotranspiration. J. Hydrol. 2021, doi:10.1016/j.jhydrol.2021.127026.

4. Hunink, J.E.; Eekhout, J.P.C.; de Vente, J.; Contreras, S.; Droogers, P.; Baille, A. Hydrological Modelling using Satellite-based Crop Coefficients: a Comparison of Methods at the Basin Scale. Remote Sens. 2017, 1-16, doi:10.3390/rs9020174.

5. Samaniego, L.; Kumar, R.; Thober, S.; Rakovec, O.; Zink, M.; Wanders, N.; Eisner, S.; Müller Schmied, H.; Sutanudjaja, E.; 
Warrach-Sagi, K.; et al. Toward seamless hydrologic predictions across spatial scales. Hydrol. Earth Syst. Sci. 2017, 21, 43234346, doi:10.5194/hess-21-4323-2017.

6. Mizukami, N.; Clark, M.P.; Newman, A.J.; Wood, A.W.; Gutmann, E.D.; Nijssen, B.; Rakovec, O.; Samaniego, L. Towards seamless large-domain parameter estimation for hydrologic models. Water Resour. Res. 2017, 53, 8020-8040, doi:10.1002/2017WR020401.

7. Conradt, T.; Wechsung, F.; Bronstert, A. Three perceptions of the evapotranspiration landscape: Comparing spatial patterns from a distributed hydrological model, remotely sensed surface temperatures, and sub-basin water balances. Hydrol. Earth Syst. Sci. 2013, doi:10.5194/hess-17-2947-2013.

8. Koch, J.; Jensen, K.H.; Stisen, S. Toward a true spatial model evaluation in distributed hydrological modeling: Kappa statistics, Fuzzy theory, and EOF-analysis benchmarked by the human perception and evaluated against a modeling case study. Water Resour. Res. 2015, doi:10.1002/2014WR016607.

9. Stisen, S.; Koch, J.; Sonnenborg, T.O.; Refsgaard, J.C.; Bircher, S.; Ringgaard, R.; Jensen, K.H. Moving beyond run-off calibration - Multivariable optimization of a surface-subsurface-atmosphere model. Hydrol. Process. 2018, 32, 2654-2668, doi:10.1002/hyp.13177.

10. Koch, J.; Demirel, M.C.; Stisen, S. The SPAtial EFficiency metric (SPAEF): multiple-component evaluation of spatial patterns for optimization of hydrological models. Geosci. Model Dev. 2018, 11, 1873-1886, doi:10.5194/gmd-11-1873-2018.

11. Zink, M.; Mai, J.; Cuntz, M.; Samaniego, L. Conditioning a Hydrologic Model Using Patterns of Remotely Sensed Land Surface Temperature. Water Resour. Res. 2018, doi:10.1002/2017WR021346.

12. Dembélé, M.; Hrachowitz, M.; Savenije, H.H.G.; Mariéthoz, G.; Schaefli, B. Improving the Predictive Skill of a Distributed Hydrological Model by Calibration on Spatial Patterns With Multiple Satellite Data Sets. Water Resour. Res. 2020, 56, e2019WR026085, doi:10.1029/2019WR026085.

13. Koch, J.; Siemann, A.; Stisen, S.; Sheffield, J. Spatial validation of large scale land surface models against monthly land surface temperature patterns using innovative performance metrics. J. Geophys. Res. Atmos. 2016, 121, 5430-5452, doi:10.1002/2015JD024482.

14. Ko, A.; Mascaro, G.; Vivoni, E.R. Strategies to Improve and Evaluate Physics-Based Hyperresolution Hydrologic Simulations at Regional Basin Scales. Water Resour. Res. 2019, doi:10.1029/2018WR023521.

15. Stisen, S.; Soltani, M.; Mendiguren, G.; Langkilde, H.; Garcia, M.; Koch, J. Spatial patterns in actual evapotranspiration climatologies for europe. Remote Sens. 2021, doi:10.3390/rs13122410.

16. Rakovec, O.; Mizukami, N.; Kumar, R.; Newman, A.J.; Thober, S.; Wood, A.W.; Clark, M.P.; Samaniego, L. Diagnostic Evaluation of Large-Domain Hydrologic Models Calibrated Across the Contiguous United States. J. Geophys. Res. Atmos. 2019, doi:10.1029/2019JD030767.

17. Koster, R.D.; Mahanama, S.P.P. Land surface controls on hydroclimatic means and variability. J. Hydrometeorol. 2012, doi:10.1175/JHM-D-12-050.1.

18. Guzinski, R.; Nieto, H.; Stisen, S.; Fensholt, R. Inter-comparison of energy balance and hydrological models for land surface energy flux estimation over a whole river catchment. Hydrol. Earth Syst. Sci. 2015, 19, 2017-2036.

19. Budyko, M.I. Climate and life; Academic Press Inc: New York, 508;

20. Greve, P.; Burek, P.; Wada, Y. Using the Budyko Framework for Calibrating a Global Hydrological Model. Water Resour. Res. 2020, doi:10.1029/2019WR026280.

21. Kushwaha, A.P.; Tiwari, A.D.; Dangar, S.; Shah, H.; Mahto, S.S.; Mishra, V. Multimodel assessment of water budget in Indian sub-continental river basins. J. Hydrol. 2021, 603, 126977, doi:https://doi.org/10.1016/j.jhydrol.2021.126977.

22. Gharari, S.; Shafiei, M.; Hrachowitz, M.; Kumar, R.; Fenicia, F.; Gupta, H. V.; Savenije, H.H.G. A constraint-based search algorithm for parameter identification of environmental models. Hydrol. Earth Syst. Sci. 2014, doi:10.5194/hess-18-4861-2014.

23. Stisen, S.; Jensen, K.H.; Sandholt, I.; Grimes, D.I.F. A remote sensing driven distributed hydrological model of the Senegal River basin. J. Hydrol. 2008, 354, 131-148, doi:10.1016/j.jhydrol.2008.03.006. 
24. Bodian, A.; Diop, L.; Panthou, G.; Dacosta, H.; Deme, A.; Dezetter, A.; Ndiaye, P.M.; Diouf, I.; Visch, T. Recent trend in hydroclimatic conditions in the Senegal River basin. Water (Switzerland) 2020, doi:10.3390/w12020436.

25. Samaniego, L.; Brenner, J.; Craven, J.; Cuntz, M.; Dalmasso, G.; Demirel, C.M.; Jing, M.; Kaluza, M.; Kumar, R.; Langenberg, B.; et al. mesoscale Hydrologic Model - mHM v5.11.1 2021.

26. Samaniego, L.; Kumar, R.; Attinger, S. Multiscale parameter regionalization of a grid-based hydrologic model at the mesoscale. Water Resour. Res. 2010, 46, doi:10.1029/2008wr007327.

27. Kumar, R.; Samaniego, L.; Attinger, S. Implications of distributed hydrologic model parameterization on water fluxes at multiple scales and locations. Water Resour. Res. 2013, 49, 360-379, doi:10.1029/2012WR012195.

28. Thober, S.; Cuntz, M.; Kelbling, M.; Kumar, R.; Mai, J.; Samaniego, L. The multiscale routing model mRM v1.0: Simple river routing at resolutions from 1 to $50 \mathrm{~km}$. Geosci. Model Dev. 2019, doi:10.5194/gmd-12-2501-2019.

29. Kumar, R.; Livneh, B.; Samaniego, L. Toward computationally efficient large-scale hydrologic predictions with a multiscale regionalization scheme. Water Resour. Res. 2013, doi:10.1002/wrcr.20431.

30. Poméon, T.; Diekkrüger, B.; Kumar, R. Computationally efficient multivariate calibration and validation of a grid-based hydrologic model in sparsely gauged West African river basins. Water (Switzerland) 2018, doi:10.3390/w10101418.

31. Dembélé, M.; Ceperley, N.; Zwart, S.J.; Salvadore, E.; Mariethoz, G.; Schaefli, B. Potential of satellite and reanalysis evaporation datasets for hydrological modelling under various model calibration strategies. Adv. Water Resour. 2020, doi:10.1016/j.advwatres.2020.103667.

32. Dembele, M.; Schaefli, B.; Van De Giesen, N. Suitability of 17 gridded rainfall and temperature datasets for large-scale hydrological modelling in West Africa. Hydrol. Earth Syst. Sci. 2020, doi:10.5194/hess-24-5379-2020.

33. Hengl, T.; Heuvelink, G.B.M.; Kempen, B.; Leenaars, J.G.B.; Walsh, M.G.; Shepherd, K.D.; Sila, A.; MacMillan, R. A.; De Jesus, J.M.; Tamene, L.; et al. Mapping soil properties of Africa at $250 \mathrm{~m}$ resolution: Random forests significantly improve current predictions. PLoS One 2015, 10, doi:10.1371/journal.pone.0125814.

34. Friedl, M.; Gray, J.; Sulla-Menashe, D. MCD12Q2 MODIS/Terra+Aqua Land Cover Dynamics Yearly L3 Global 500m SIN Grid V006.

35. Myneni, R.B.; Knyazikhin, Y.; Park, T. MCD15A2H MODIS/Terra+Aqua Leaf Area Index/FPAR 8-day L4 Global 500m SIN Grid V006 Available online: https://doi.org/10.5067/MODIS/MCD15A2H.006 (accessed on Aug 6, 2021).

36. Hersbach, H.; Bell, B.; Berrisford, P.; Hirahara, S.; Horányi, A.; Muñoz-Sabater, J.; Nicolas, J.; Peubey, C.; Radu, R.; Schepers, D.; et al. The ERA5 global reanalysis. Q. J. R. Meteorol. Soc. 2020, doi:10.1002/qj.3803.

37. George H. Hargreaves; Zohrab A. Samani Reference Crop Evapotranspiration from Temperature. Appl. Eng. Agric. 1985, doi:10.13031/2013.26773.

38. Funk, C.; Peterson, P.; Landsfeld, M.; Pedreros, D.; Verdin, J.; Shukla, S.; Husak, G.; Rowland, J.; Harrison, L.; Hoell, A.; et al. The climate hazards infrared precipitation with stations - A new environmental record for monitoring extremes. Sci. Data 2015, doi:10.1038/sdata.2015.66.

39. Funk, C.C.; Peterson, P.J.; Landsfeld, M.F.; Pedreros, D.H.; Verdin, J.P.; Rowland, J.D.; Romero, B.E.; Husak, G.J.; Michaelsen, J.C.; Verdin, A.P. A Quasi-Global Precipitation Time Series for Drought Monitoring. U.S. Geol. Surv. Data Ser. 2014.

40. Running, S.; Mu, Q.; Zhao, M. MOD16A2 MODIS/Terra Net Evapotranspiration 8-Day L4 Global 500m SIN Grid V006.

41. Gupta, H. V; Kling, H.; Yilmaz, K.K.; Martinez, G.F. Decomposition of the mean squared error and NSE performance criteria: Implications for improving hydrological modelling. J. Hydrol. 2009, 377, 80-91, doi:http://dx.doi.org/10.1016/j.jhydrol.2009.08.003.

42. Matott, L.S. OSTRICH: an Optimization Software Tool, Documentation and User's Guide. Univ. Buffalo Cent. Comput. Res. 2017, Version 17, 79.

43. Tolson, B.; Sharma, V.; Swayne, D.A. Parallel Implementations of the Dynamically Dimensioned Search (DDS) Algorithm. In Proceedings of the 7th International Symposium on Environmental Software Systems; 2007.

44. Demirel, M.C.; Koch, J.; Mendiguren, G.; Stisen, S. Spatial pattern oriented multicriteria sensitivity analysis of a distributed 
hydrologic model. Water (Switzerland) 2018, doi:10.3390/w10091188. 